\title{
Editorial
}

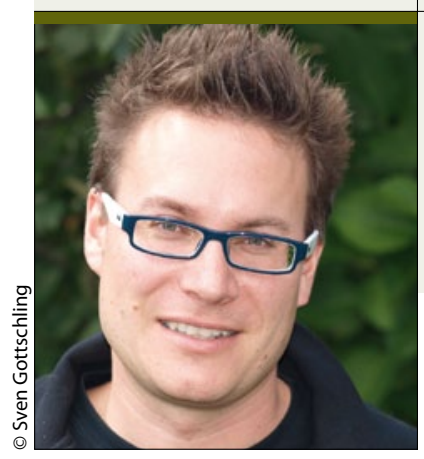

Trotz des seit 2007 bestehenden Rechtsanspruchs können selbst optimistisch geschätzt höchstens 20\% aller Familien auf SAPV

für ihre lebenslimitierend erkrankten Kinder hoffen.

PD Dr. Sven Gottschling,

Zentrum für Palliativmedizin und Kinderschmerztherapie,

Universitätsklinikum des Saarlandes

\section{SAPV für Kinder - Anspruch und Wirklichkeit}

$\mathrm{F}$ assungslos halten Eltern ein Schreiben ihrer Krankenkasse in der Hand. Sie schreibt: „Gemäß MDK-Gutachten erfüllt Ihr Kind die Anspruchsvoraussetzungen für eine SAPV-Versorgung nicht. Ihr Kind leidet nicht unter einer lebenslimitierenden Erkrankung." Wenige Stunden zuvor war ihr 4-jähriger Sohn nach wochenlanger intensiver Versorgung durch ein Kinder-SAPV-Team zu Hause an den Folgen einer Stoffwechselerkrankung verstorben. Krampfanfälle, Schmerzkrisen und Luftnotattacken hatten sowohl die Eltern als auch die Profis in den letzten Wochen sehr gefordert. Was wie ein schlechtes Drehbuch klingt, ist leider bittere Realität für viele betroffene Familien. Besonders, wenn es sich nicht um die todesnahe Versorgung krebskranker Kinder handelt, wird der spezifische SAPV-Bedarf des Kindes von Kostenträgern und MDK oft nicht verstanden.

Aus Untersuchungen der letzten Jahre ist bekannt, dass Kinder mit lebenslimitierenden Erkrankungen schlechter versorgt sind, leidvoller leben und auch sterben als erwachsene Patienten. Hat sich hier in den vergangenen Jahren etwas verändert? Obgleich seit 2007 ein Rechtsanspruch besteht, können - optimistisch geschätzt - höchstens $20 \%$ aller Familien auf SAPV für ihre erkrankten Kinder hoffen. Und bestehende Teams sind hoffnungslos unterfinanziert, neue werden daher erst gar nicht gegründet. Seit über einem Jahr kämpfen wir Kinderpalliativ-Versorger darum, bundesweit eine flächen- aber auch kostendeckende Versorgung der betroffenen Kinder durchzusetzen.

Inmitten dieses Prozesses veröffentlichte der GKVSpitzenverband am 05.11.2012 die neuen Empfehlungen für die spezialisierte ambulante Palliativversorgung, deren Inhalt mich fassungslos macht: So enthalten die Empfehlungen z. B. Schätzungen von Experten zum SAPV-Bedarf für Erwachsene. Es folgt dann der lapidare Satz: für Kinder und Jugendliche lag keine gesonderte Schätzung vor. Die Verantwortlichen hät- ten sich aber die Mühe machen dürfen, danach zu suchen oder entsprechende Experten zu befragen denn diese Erhebungen liegen selbstverständlich vor.

Der eigentliche Gipfel des GKV-Papiers ist jedoch die Aussage, dass in SAPV-Teams, die sowohl Erwachsene als auch Kinder versorgen, mindestens ein Arzt für Kinder und Jugendmedizin mit Palliativ-Qualifikation arbeiten muss. Dies wird im Folgenden nicht näher spezifiziert. Hiermit wurde eine Öffnungsklausel geschaffen, die es faktisch jedem ErwachsenenSAPV-Team ermöglicht, Kinder und Jugendliche auch ohne spezielle Qualifikation zu versorgen, solange ein Kinder-Palliativ-Mediziner und eine Kinderkrankenpflegekraft z. B. auf 400-Euro-Basis bereit sind, einem Erwachsenen-SAPV-Team ihre Namen zur Verfügung zu stellen.

Ich muss daher feststellen, dass eine auf die Bedürfnisse von Kindern und Jugendlichen abgestimmte SAPV-Versorgung durch entsprechend qualifizierte Kinderärzte und Kinderkrankenpflegekräfte gemäß dem Motto: „die paar Kinder können die Erwachsenen-Teams doch auch mitversorgen" schlichtweg nicht gewünscht ist. Bislang fanden die besonderen Belange von Kindern in der SAPV keine adäquate Berücksichtigung. In den neuen Empfehlungen des GKV-Spitzenverbandes mit der Aufweichung kinderspezifischer SAPV-Strukturen wird nun sogar in Zweifel gezogen, dass lebenslimitierend erkrankte Kinder und Jugendliche überhaupt besondere Bedürfnisse in der Versorgung haben. Es ist in meinen Augen mehr als überfällig, dass die Politik ihrer Verpflichtung nachkommt, endlich den seit fünf Jahren bestehenden Rechtsanspruch auf SAPV in aller Konsequenz auch und gerade für Kinder durchzusetzen.

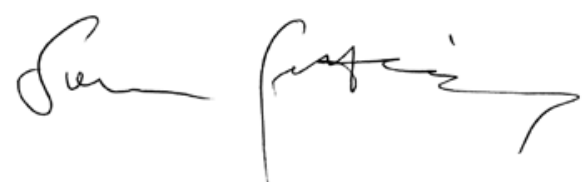

